\title{
Melting of polydisperse hard disks
}

\author{
Sander Pronk and Daan Frenkel \\ FOM Institute for Atomic and Molecular Physics, Kruislaan 407, 1098 SJ Amsterdam, The Netherlands
}

(Received 27 October 2003; revised manuscript received 2 February 2004; published 15 June 2004)

\begin{abstract}
The melting of a polydisperse hard-disk system is investigated by Monte Carlo simulations in the semigrand canonical ensemble. This is done in the context of possible continuous melting by a dislocation-unbinding mechanism, as an extension of the two-dimensional hard-disk melting problem. We find that while there is pronounced fractionation in polydispersity, the apparent density-polydispersity gap does not increase in width, contrary to 3D polydisperse hard spheres. The point where the Young's modulus is low enough for the dislocation unbinding to occur moves with the apparent melting point, but stays within the density gap, just like for the monodisperse hard-disk system. Additionally, we find that throughout the accessible polydispersity range, the bound dislocation-pair concentration is high enough to affect the dislocation-unbinding melting as predicted by Kosterlitz, Thouless, Halperin, Nelson, and Young.
\end{abstract}

DOI: 10.1103/PhysRevE.69.066123

PACS number(s): 64.60.Fr, 64.70.Dv, 05.10.Ln

\section{INTRODUCTION}

In 1930s, Landau and Peierls showed that twodimensional (2D) solids are qualitatively different from their 3D counterpart, as they lack long-ranged positional order (see, e.g., Ref. [1]). However, 2D crystals do have longranged bond-orientational order and, in this respect, they differ from the isotropic liquid phase where both translational and bond-orientational order are short ranged.

In 1970s, Kosterlitz and Thouless suggested that melting of two-dimensional crystals may be quite different from 3D melting. In particular, they proposed that melting in two dimensions may proceed via a continuous dislocationunbinding transition.

Kosterlitz and Thouless [2,3] showed that the free energy associated with a single dislocation becomes negative when

$$
K<16 \pi k_{B} T,
$$

where $K$ is the Young's modulus of the crystal. At the point where $K=16 \pi k_{B} T$, dislocation pairs can unbind and, as solid with free dislocations "flow" under shear, Kosterlitz and Thouless interpreted this temperature as the melting point. In a more detailed analysis, Halperin and Nelson [2] and Young [4] showed that dislocation unbinding is not enough to complete the melting process. At the point where the condition of Eq. (1) is first satisfied, the system undergoes a (continuous) transition from a $2 \mathrm{D}$ crystal to a hexatic phase. The hexatic phase is characterized by short-ranged (exponentially decaying) positional order, but quasi-long-ranged (algebraically decaying) orientational order: the positional order is destroyed by the presence of the unbound dislocations. A second (continuous) phase transition is required to transform the hexatic phase into an isotropic liquid with short-ranged bond-orientational order.

The Kosterlitz-Thouless-Halperin-Nelson-Young (KTHNY) theory makes precise predictions about the behavior of the correlation functions of both the translational and orientational order parameters. It should be stressed, however, that the KTHNY theory describes only a possible scenario: it is also possible that one or both of the continuous transitions are of first order, and even that there is a direct first-order transition from the crystal to the isotropic fluid.

The KTHNY predictions sparked off an intensive search for real or model systems that would exhibit this two-stage melting process (for an early review, see Ref. [5]. More recent examples can be found in Refs. [6-9]).

Surprisingly, however, there is still no satisfactory answer to the question whether the KTHNY scenario applies even to the simplest of all two-dimensional model systems, namely, hard, elastic disks. In fact, this system was the very first to be studied in any computer simulation [10].

The reason why it is difficult to determine the nature of the melting transition is that finite-size effects tend to obscure the distinction between first order and continuous melting in 2D systems (see Ref. [5]).

In the case of hard disks, the early work by Alder and Wainwright suggested that the hard-disk melting transition was of first order [11] (in the very early work of Metropolis et al. [10], the computing power was insufficient to draw meaningful conclusions about the nature of the melting transition). The hard-disk melting problem was revisited many times after the suggestion of the KTHNY scenario, but the evidence is still ambiguous. Evidence for continuous melting was reported in Ref. [12], while evidence for a first-order phase transition was presented in Refs. [13-16]. In addition, several publications could not distinguish between the two scenarios [17-19]. More recently, there has been some evidence for the KTHNY scenario [20-22] but the matter still seems far from settled.

One possible route to tackle this problem would be to consider hard disks as a special case of a more general class of systems, and study possible trends in the phase behavior of this generalized model. In the present case, we consider the 2D hard-disk system as a special case of polydisperse disks. In 3D hard spheres, the melting transition is of first order. As the polydispersity is increased the difference in volume fraction of the coexisting solid and liquid phases widens with increasing polydispersity $[23,24]$. One might expect similar behavior in two dimensions if the solid-liquid transition would be of first order. 
While polydispersity in two-dimensional systems has been studied before, it was in the context of melting by increasing size dispersity for Lennard-Jones systems [25] or in the context of a possible glass transition [26,27]. In the present paper, we examine the phase behavior of polydisperse hard-disk systems.

\section{THE SYSTEM}

\section{A. The semigrand canonical ensemble}

The model for polydispersity is based on the semigrand canonical ensemble [28]; this ensemble has previously been used to study the phase diagram of polydisperse 3D hard spheres $[23,24]$. The semigrand canonical ensemble can be seen as a hybrid version of the canonical ensemble and the grand canonical ensemble. It is characterized by a thermodynamic potential $X$ that satisfies the following fundamental thermodynamic relation [29]:

$$
d X=-S d T-P d V-\int N(\sigma) \delta \mu(\sigma) d \sigma .
$$

Here $S$ is the entropy of the system, $T$ the temperature, $P$ the pressure, and $V$ the volume. $N(\sigma) d \sigma$ denotes the number of particles with diameter between $\sigma$ and $\sigma+d \sigma$ and $\mu(\sigma)$ is the chemical potential of particles with diameter $\sigma$. We now add and subtract a term containing the chemical potential of a reference species $\sigma_{0}$ from the complete differential:

$$
d X=-S d T-P d V-N d \mu\left(\sigma_{0}\right)-\int N(\sigma) \delta \Delta \mu(\sigma) d \sigma,
$$

where we replace $\mu(\sigma)-\mu\left(\sigma_{0}\right)$ with $\Delta \mu(\sigma)$. We now perform a Legendre transformation to a new ensemble that has $N$ as a thermodynamic control parameter instead of $\mu\left(\sigma_{0}\right)$ (and $P$ instead of $V$ ):

$$
d Y=-S d T+V d P+\mu\left(\sigma_{0}\right) d N-\int N(\sigma) \delta \Delta \mu(\sigma) d \sigma,
$$

which, in explicit form, becomes (with the Euler equation)

$$
\begin{aligned}
Y(N, \Delta \mu(\sigma), P, T)= & U-T S+P V+N \mu\left(\sigma_{0}\right) \\
& -\int N(\sigma) \Delta \mu(\sigma) d \sigma \\
= & N \mu\left(\sigma_{0}\right) .
\end{aligned}
$$

The partition sum for this ensemble is

$$
\begin{aligned}
\Upsilon & (N, \Delta \mu(\sigma), P, T) \\
& =\int d \sigma^{N} \int d V \int d s^{N} \exp \left(-\beta\left\{P V+U\left(V, s^{N}\right)\right.\right. \\
& -\Delta \mu(\sigma) N(\sigma)]\},
\end{aligned}
$$

with $Y=-k_{B} T \ln Y$. For simulation purposes, the semigrand canonical ensemble can be interpreted as the one where there is constant number of particles that can change identity. This identity switching can be an extra move in a Monte Carlo simulation; in a polydisperse mixture, this would amount to a particle size change with an acceptance criterion based on the functional form for the chemical potential $\Delta \mu(\sigma)$.

As in Ref. [23], we use the following functional form for the chemical potential:

$$
\Delta \mu(\sigma)=-k_{B} T \frac{\left(\sigma-\sigma_{0}\right)^{2}}{2 \nu^{2}},
$$

which, at zero density, will give a Gaussian particle size distribution according to the partition sum of Eq. (6). In practice, the size distribution is Gaussian-like at the densities of the crystalline phase.

\section{B. Order parameters}

For the study of the properties of the phases and the phase transitions, some order parameters have been used, which are standard in the studying of 2D melting [5] and for which the KTHNY melting scenario makes explicit predictions [2]. We define the $n$-fold bond-orientational order at $\mathbf{x}_{i}$, the position of particle $i$, as

$$
\psi_{n}\left(\mathbf{x}^{i}\right)=\frac{1}{N_{i}} \sum_{j=1}^{N_{i}} e^{i n \theta_{j}\left(\mathbf{x}^{i}\right)},
$$

where $N_{i}$ is the number of neighbors and $\theta_{j}\left(\mathbf{x}^{i}\right)$ is the angle between an arbitrary (fixed) axis and the line connecting particle $i$ with its $j$ th neighbor; two particles are neighbors if they share a Voronoi cell edge. For systems that tend to crystallize into triangular lattices, the leading bond-order parameter is the one for which $n=6$. The global value of the order parameter is simply the mean of the local values.

The positional order is measured using the static structure factor $S(\mathbf{q})$ at one specific scattering vector $\mathbf{q}$ equal to a reciprocal lattice vector of a perfect crystal with orientation and lattice spacing taken from the system. To check for hexagonal crystalline positional order, the lattice vector $a_{0}$ is set to its ideal value for a given packing fraction:

$$
a_{0}=\left(\frac{\pi / \sqrt{12}}{\eta}\right)^{1 / 2} \text {. }
$$

The crystal orientation is taken from the mean angle obtained from the global hexagonal bond-orientational order parameter:

$$
\Psi_{6}=\frac{1}{N} \sum_{i=1}^{N} \psi_{6}\left(\mathbf{x}_{i}\right),
$$

which specifies the orientation of one of the six equivalent crystal axes within an angular range $0 \leqslant \alpha<\pi / 3$. Once the average orientation of the nearest-neighbor "bonds" $\mathbf{a}_{0}$ has been specified, it is straightforward to deduce the orientation of the corresponding reciprocal lattice vector $\mathbf{G}$ through $\mathbf{G} \cdot \mathbf{a}_{0}=2 \pi$. The positional order parameter $\zeta$ of the crystal is then given by

$$
\zeta\left(\mathbf{x}_{i}\right)=e^{i \mathbf{G} \cdot \mathbf{x}_{i}} .
$$

Radial correlation functions of the order parameters are defined as 


$$
\begin{gathered}
g_{6}(r)=\left\langle\psi_{6}^{*}(\mathbf{0}) \psi_{6}(r)\right\rangle / g(r), \\
\zeta(r)=\left\langle\zeta^{*}(\mathbf{0}) \zeta(r)\right\rangle / g(r) .
\end{gathered}
$$

In two-dimensional systems, $\zeta$ is expected to decay to zero, either exponentially (short ranged order) or algebraically (quasi-long-ranged). The KTHNY melting scenario makes predictions for the decay of the orientational order in the hexatic and phase: $g_{6}(r) \sim r^{-\eta_{6}}$ with $\eta_{6} \rightarrow \frac{1}{4}$ at the melting of the hexatic phase into the liquid phase [2,5].

\section{Elasticity}

Equation (1) provides a very useful test to decide whether or not a 2D melting can be of the KTHNY type. If we find that Young's modulus drops below the "magical" value of $16 \pi k_{B} T$ in an otherwise stable solid, then it is very likely that this solid melts by dislocation unbinding. Conversely, if we find that this magical value is only crossed at densities where we know that the isotropic liquid phase is thermodynamically stable, then it is reasonable to assume that melting is a first-order transition. Often, however, the simulations do not provide a clear answer, as the point where $K=16 \pi k_{B} T$ is located in the intermediate density regime that may either be a two-phase region separating two stable phase or the domain of the elusive hexatic phase.

The Young's modulus is defined through the shear $\left(\lambda_{L}\right)$ and bulk $\left(\mu_{L}\right)$ Lamé elastic constants in 2D:

$$
K=\frac{4 a_{0}^{2} \mu_{L}\left(\mu_{L}+\lambda_{L}\right)}{2 \mu_{L}+\lambda_{L}} \leqslant 16 \pi,
$$

where $a_{0}$ is the equilibrium lattice spacing. The Lamé elastic constants are related to the second-order elastic constants (i.e., the elastic constants defined by the second derivative of the free energy to the Lagrangian strain) through

$$
\begin{aligned}
& C_{11}=\lambda_{L}+2 \mu_{L}, \\
& C_{12}=\lambda_{L}, \\
& C_{44}=\mu_{L}-P .
\end{aligned}
$$

The theory of the KTHNY scenario is based on a group renormalization of both the Young's modulus and the dislocation fugacity

$$
y=e^{-\beta E_{c}},
$$

where $E_{c}$ is the core energy of a dislocation. As the length scale of the renormalization is increased towards infinity, the dislocation fugacity and the elastic constants that constitute $K$ tend to their "renormalized" values, where the (longrange) effect of (presumably low-concentration) dislocations is properly taken into account.

The elastic constants measured in this work, although calculated at finite sizes, should be close to their renormalized values because of the high concentration of dislocations in the hard-disk system, as will be shown in Sec. III.

When specifying elastic constants of a polydisperse system, we should distinguish between "quenched" and "an- nealed" elastic constants. Quenched elastic constants measure the second strain derivative of the free energy of a polydisperse crystal with a "frozen" size distribution; i.e., the particle size distribution is assumed not to respond to the deformation. In contrast, the "annealed" elastic constants measure the second strain derivative of the semigrand potential. In this case, the particle size distribution is assumed to respond to the deformation. The quenched constants are the ones that are presumably measured in mechanical experiments that probe the elastic deformation of a polydisperse solid. But the annealed constants describe the equilibrium state of a deformed polydisperse solid. In order to determine the critical value for Young's modulus in a polydisperse 2D solid, we computed the annealed elastic constants, as these determine the equilibrium behavior of the system.

To calculate the elastic constants at different polydispersities, a hybrid Monte Carlo-molecular dynamics method was used, where Monte Carlo runs sampling particle diameters and positions in the strained system were followed by molecular dynamics runs where the stress tensor was measured using the instantaneous realization of the particle size distribution. The algorithm thus effectively calculates the elastic constants of many realizations of a polydisperse hardsphere crystal.

The quenched elastic constants will be larger than the annealed quantities, because of the concavity of the free energy. Hence the corresponding quenched Young's modulus will only reach the instability limit $K=16 \pi$ at lower densities.

The strains used in the elastic constant determination were

$$
\begin{aligned}
\alpha_{i j}^{1} & =\left(\begin{array}{cc}
1+a & 0 \\
0 & 1+a
\end{array}\right), \\
\alpha_{i j}^{2} & =\left(\begin{array}{ll}
1 & b \\
0 & 1
\end{array}\right),
\end{aligned}
$$

with $a$ as the (small) strain parameter. The resulting stress derivatives give us [30]

$$
\begin{aligned}
& \frac{d T_{11}}{d a}=\frac{\partial T_{11}}{\partial \alpha_{11}^{1}}+\frac{\partial T_{11}}{\partial \alpha_{22}^{1}}=C_{11}+C_{12}=2 \lambda_{L}+2 \mu_{L}, \\
& \frac{d T_{12}}{d b}=\frac{\partial T_{12}}{\partial \alpha_{12}^{1}}=C_{44}+P=\mu_{L},
\end{aligned}
$$

allowing us to calculate the Young's modulus of Eq. (14).

\section{SIMULATIONS}

The simulations were performed on systems containing $59 \times 68=4012$ particles, with a chemical potential distribution width (polydispersity) parameter $\nu$ [see Eq. (7)] varying between $\nu=0.00025$ and $\nu=0.008$. For higher values of $\nu$ (higher polydispersities), the equilibration was exceedingly slow, even with the combined volume-particle radius sampling: equilibration took $1.3 \times 10^{6}-2.5 \times 10^{6}$ Monte Carlo (MC) steps per particle and data were sampled during 1 $\times 10^{6}$ steps per particle; around $20 \%$ of steps were devoted 


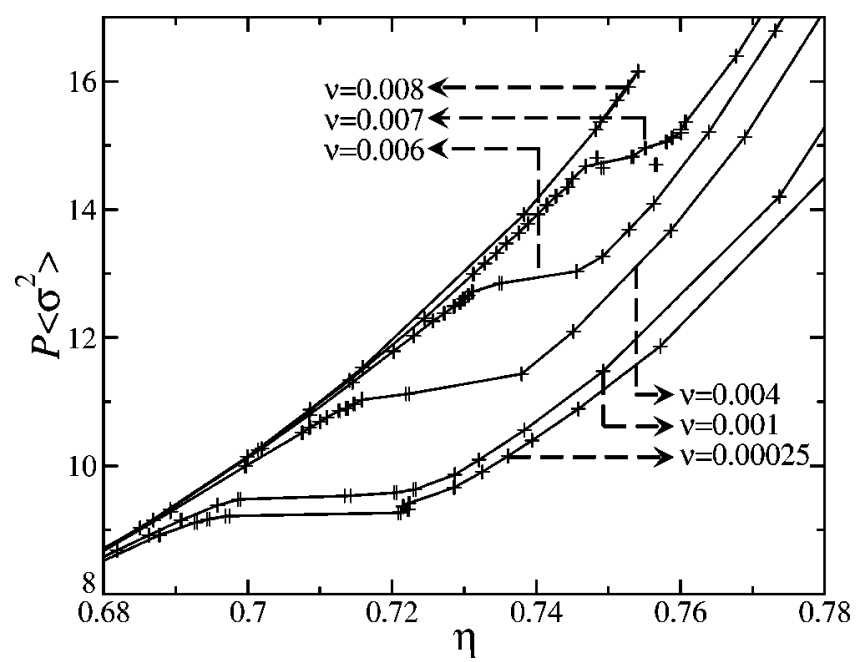

FIG. 1. Calculated equations of state (with normalized pressure) for varying polydispersity control parameter $\nu$. System size is 4012 particles.

to particle radius steps. For $N=4012$, the simulation time was $6-8 \mathrm{~h}$ on an Athlon $1600+\mathrm{CPU}$.

Monte Carlo simulations of the semigrand canonical ensemble of Eq. (6) involve displacement moves, volume moves, and identity change moves, which in the current context mean particle radius moves. To speed up the simulation, the coupling between mean particle radius and system size is exploited to remove the global volume moves and simultaneous system + particle size scaling was introduced. This improves the statistics of sampling considerably [23]. It turns out that, in practice, a real simultaneous system + particle size scaling is at least as fast as the analytical integration of the scaling part of the partition sum also described in Ref. [23].

The resulting equations of state are shown in Fig. 1. From this figure, it is clear that the phase transition, which is around packing fraction $\eta \approx 0.70$ for the monodisperse case [17-19], shifts to higher packing fractions and higher pressures with increasing polydispersity. At $\nu=0.008$, the system cannot be made to freeze at all. A similar phenomenon was observed in Ref. [23] - it is due to the choice of the functional form for the chemical potential [Eq. (7)]. In addition, for $\nu=0.007$, it is extremely difficult to equilibrate the system properly in the vicinity of the phase transition.

As is the case for $3 \mathrm{D}$ spheres, the liquid branches of the equations of state very nearly superimpose [23]. However, near the phase transition, the pressure appears to increase slightly with polydispersity. Upon further compression, the system undergoes a phase transition with an apparent density gap. However, because of the relatively small system size, the presence of such an apparent density gap is also compatible with continuous melting. We find that the density gap decreases with increasing polydispersity.

In Fig. 2 we plot the variation in polydispersity upon freezing. As a measure for the polydispersity, we use $s$ $\equiv\left\langle\sigma^{2}\right\rangle /\langle\sigma\rangle^{2}-1$. In the same figure, the apparent density gap is also shown (the borders of the density gap shown here are simply the solid with the lowest packing fraction and the liquid with the highest packing fraction found in the simula-

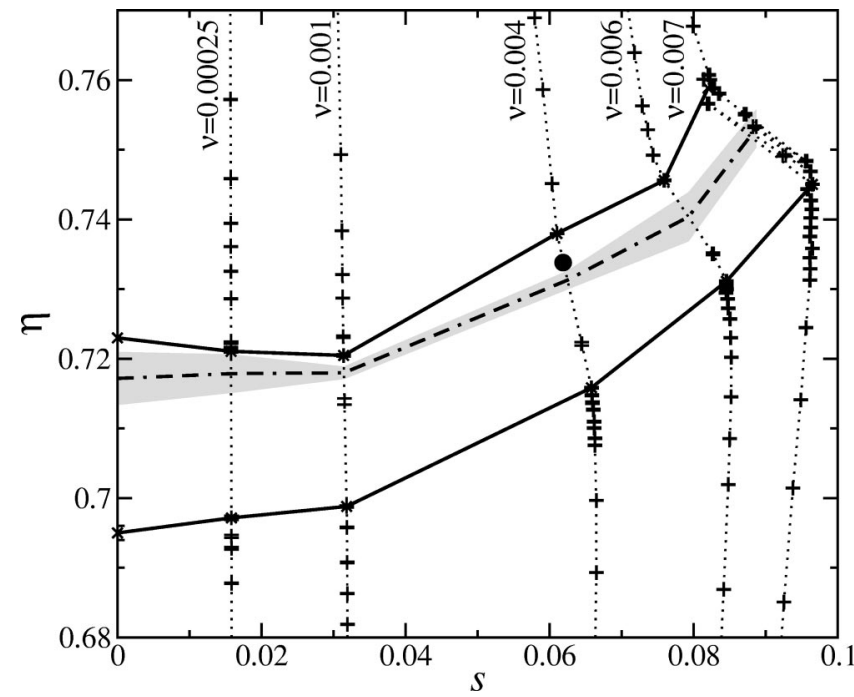

FIG. 2. Packing fraction $\eta$ as a function of polydispersity $s$ for varying $\nu$, with $N=4012$. The boundaries of the apparent density gap are shown as the solid lines; the density gap of the monodisperse $(s=0)$ case is taken from data by Jaster [18], with $N=4096$. The dashed line shows the location of packing fractions (with matching polydispersities) extrapolated to be $K=16 \pi$; the gray area denotes an estimate of the size of the error. The black circle shows the location of the $K=16 \pi$ point obtained using a larger system size $(N=4012)$ for the elasticity simulations.

tions). As in the 3D case $[23,24]$, the freezing point moves to higher volume fractions as the polydispersity is increased and size fractionation is also increased. But, whereas the density gap widens upon freezing in 3D, it appears, if anything, to decrease in 2D (except for the case of $\nu=0.007$, which seems to be poorly equilibrated). Additionally, the maximum polydispersity at which the solid seems to remain stable (around $8 \%$ ) is considerably higher than in 3D $(5.7 \%)$.

In the semigrand canonical ensemble, the system equilibrates to a mean particle size, dependent on pressure and $\nu$. Figure 3 shows this mean particle size as a function of packing fraction: the phase transitions show up as jumps in the packing fraction, but not in the mean particle size. This means that there is no particle size fractionation (the mean of the particle diameter distribution does not change) while there is, as can be seen in Fig. 2, polydispersity fractionation (the width of the particle diameter distribution changes).

An example of the behavior of the order parameters near the phase transition is shown in Fig. 4. The positional and orientational order both increase sharply (but not quite simultaneously) in the region of the phase transition, similar to what one finds for monodisperse hard disks. Although the decay of the orientational correlation function $g_{6}(r)$ goes with $r^{-1 / 4}$, as predicted by the KTHNY scenario for the hexatic at the hexatic-crystal transition, this seems to be a coincidence, because a further simulation of the same configuration yields a different (but still algebraic) decay rate. This indicates that the typical decay "time" of fluctuations in the system exceeds the typical length of the (rather long) simulations.

We stress that all observations reported thus far are consistent with either a KTHNY scenario or a weak first-order 


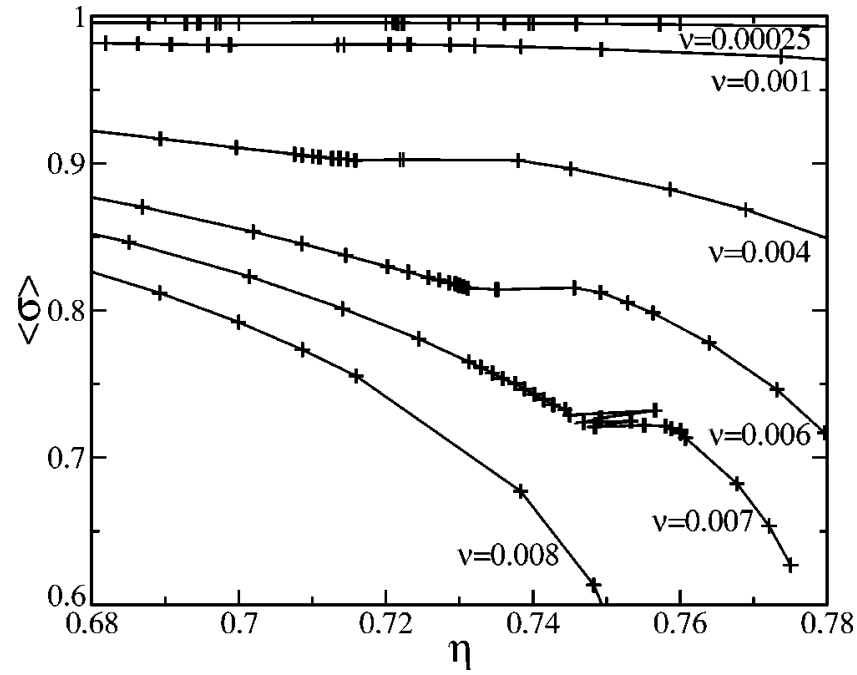

FIG. 3. Mean particle diameter $\langle\sigma\rangle$ as a function of packing fraction $\eta$ for the different values of the polydispersity control parameter $\nu$.

transition. The KTHNY scenario, however, is based on the assumption that the concentration of bound dislocations in the crystal phase is low; dislocation interaction is not taken into account and the Kosterlitz-Thouless normalization is based on an expansion in the dislocation-unbinding length which may be unrealistically long for high dislocation concentrations. We measured the concentration of bound dislocation pairs (see Fig. 5). In the figure, we show the concentration of seven coordinated particles. Because in the crystal the number of eight or more coordinated particles is negligible, and as the number of point defects turns out to be an order of magnitude lower than the number of bound dislocations [19], the number of seven coordinated particles is a good measure of the dislocation count. At the melting point (the boundary of the apparent density gap) the dislocation concentration varies from $1 \%$ to more than $3 \%$. As the concentration of dislocation pairs depends sensitively on the dislocation core energy, this suggests that the core energy is rather low. Note that a core energy less than $(2-4) k_{B} T$ is not compatible with KTHNY melting [31,32].

Neglecting the dislocation-dislocation interaction (a very crude approximation), but including the internal elastic energy and entropy of dislocation pairs [20,33], this defect den-

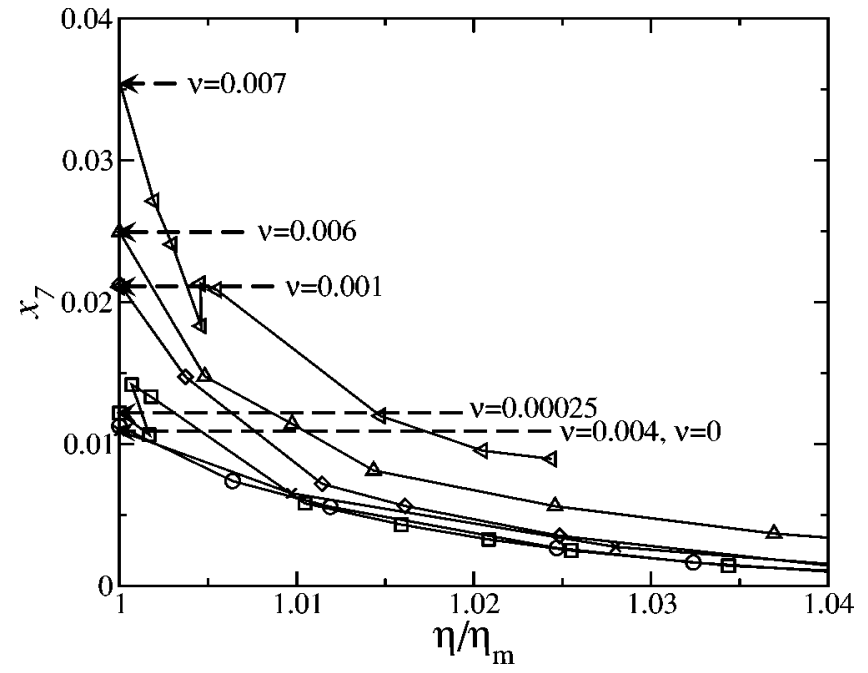

FIG. 5. Concentration of seven coordinated particles $x_{7}$ (a measure for the number of dislocations) as a function of packing fraction relative to the melting packing fraction (determined by the boundaries of the apparent density gap).

sity would be consistent with a core energy of approximately $5 k_{B} T$ over the full polydispersity range. This, however, is a crude approximation and the real core energy may be several $k_{B} T$ off. The value is compatible with both the value of $6 k_{B} T$ given in Ref. [20] and the value of $11 k_{B} T$ at a much higher packing fraction of 0.82 calculated in Ref. [34].

\section{ELASTIC CONSTANTS}

The elastic constants were measured using the method described in Sec. II C, using simulations of 412 particles, equilibrating for $4 \times 10^{6} \mathrm{MC}$ steps and $4 \times 10^{4}$ molecular dynamics (MD) collisions per particle and measuring up 5 $\times 10^{6} \mathrm{MC}$ steps and $1.5 \times 10^{6} \mathrm{MD}$ collisions per particle. Earlier work [19] had shown that the elastic constants are not significantly affected by the presence of point defects such as vacancies. Away from the KTHNY transition, the elastic constants are not very sensitive to finite-size effects [14]. Of course, this is not true close to a KTHNY transition, but this will turn out to be less relevant for the present system because we always observe melting before we get into the "danger zone."

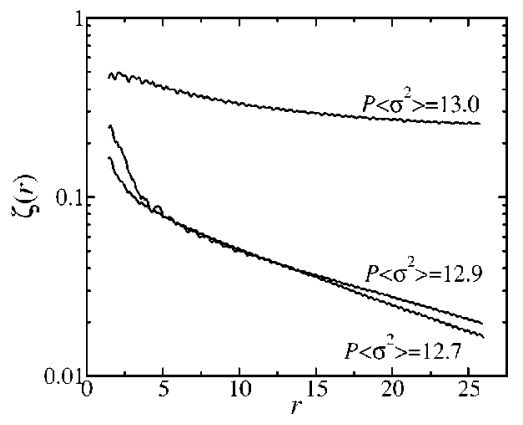

FIG. 4. Example of the decay of the orientational (left) and positional (right) order parameters over the phase transition for $\nu=0.006$. The decay of the positional order parameter, $g_{6}(r)$, goes as $g_{6}(r) \propto r^{-1 / 4}$ at the intermediate pressure $P\left\langle\sigma^{2}\right\rangle=12.7$. The positional order parameters decay exponentially for both lower pressures. 


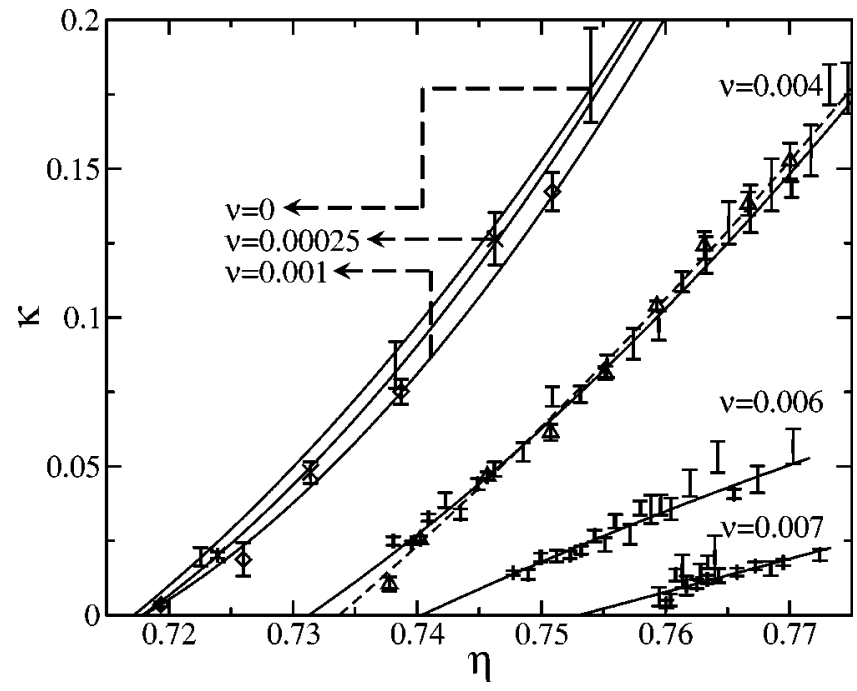

FIG. 6. Fractional exponent of the Young's modulus $\kappa=(1$ $-16 \pi / K)^{\nu_{\mathrm{KT}}}$ with $\nu_{\mathrm{KT}}=0.3696$ for the different polydispersities, as a function of packing fraction. The lines are the fits to determine the locations of $K=16 \pi$. The points at $\nu=0.004$ with the triangles together with the fit shown with the dashed line are results of calculations done with 4012 particles.

The results of the simulations are shown in Fig. 6; here an exponential of the Young's modulus $\kappa=(1-16 \pi / K)^{\nu_{\mathrm{KT}}}$, where $\nu_{\mathrm{KT}}$ is the Kosterlitz-Thouless renormalization exponent, which has a value of $\nu_{K T} \approx 0.36963$ [2]. This form is chosen because close to the KTHNY transition, the Young's modulus - expressed as function of the difference between the packing fraction $\eta$ and the packing fraction at the KTHNY, $\eta_{\mathrm{KT}}$-behaves as [5]

$$
\frac{K}{16 \pi}=\frac{1}{1-c\left(\eta-\eta_{\mathrm{KT}}\right)^{\nu_{\mathrm{KT}}}} .
$$

The measured $\kappa$ values are then fitted to a second-order polynomial. The results for the monodisperse case $(\nu=0)$ are similar to those by Wojciechowski et al. [14,35] and by Bates and Frenkel [19].

To check for finite-size effects in the elastic constants calculations, the calculations for $\nu=0.004$ were also done with 4012 particles. The results are shown in Fig. 6 and in Fig. 2; the $K=16 \pi$ extrapolation is very similar to the smaller 412 particle system and agrees within the error margin.

From the locations of the $K=16 \pi$ line in Fig. 2 it is clear that the situation with respect to the type of phase transition is, even for higher polydispersities, similar to that of the monodisperse system; the points seem to follow-within the statistical uncertainties - not only the loci of the phase transitions, but also the position within the density gap.

\section{CONCLUSION}

In this paper, we explored the effect of polydispersity on the nature of the $2 \mathrm{D}$ melting transition is a system of $2 \mathrm{D}$ hard disks.

We find that the solid-liquid phase transition shifts to higher packing fractions as the polydispersity increases, and that polydispersity fractionation takes place in the region of the phase transition. The maximum polydispersity at which the solid can be stable is larger than in 3D hard spheres.

The density-polydispersity gap, be it real or apparent, does not seem to increase in size with increasing polydispersity. The fact that the points for which $K=16 \pi$ appear to be located in the two-phase region supports the assumption that the melting transition is of first order. Even if this should not be the case, the high dislocation concentration will presumably have an effect on the KTHNY predictions.

\section{ACKNOWLEDGMENTS}

The work of the FOM institute is part of the research program of the Foundation for Fundamental Research on Matter (FOM) and was made possible through financial support by the Dutch Foundation for Scientific Research (NWO).
[1] R. Peierls, Surprises in Theoretical Physics (Princeton University Press, Princeton, 1979).

[2] D. R. Nelson, Defects and Geometry in Condensed Matter (Cambridge University Press, Cambridge, 2002).

[3] P. M. Chaikin and T. C. Lubensky, Principles of Condensed Matter Physics (Cambridge University Press, Cambridge, 1995).

[4] A. P. Young, Phys. Rev. B 19, 1855 (1979).

[5] K. J. Strandburg, Rev. Mod. Phys. 60, 161 (1988).

[6] K. Bagchi, H. C. Andersen, and W. Swope, Phys. Rev. Lett. 76, 255 (1996).

[7] K. Chen, T. Kaplan, and M. Mostoller, Phys. Rev. Lett. 74, 4019 (1995).

[8] P. Bladon and D. Frenkel, Phys. Rev. Lett. 74, 2519 (1995).

[9] K. Zahn, R. Lenke, and G. Maret, Phys. Rev. Lett. 82, 2721 (1999).
[10] N. Metropolis, A. W. Rosenbluth, M. N. Rosenbluth, A. H. Teller, and E. Teller, J. Chem. Phys. 21, 1087 (1953).

[11] B. J. Alder and T. E. Wainwright, Phys. Rev. 127, 359 (1961).

[12] J. F. Fernández, J. J. Alonso, and J. Stankiewicz, Phys. Rev. Lett. 75, 3477 (1995).

[13] J. Lee and K. J. Strandburg, Phys. Rev. B 46, 11190 (1992).

[14] K. W. Wojciechowski and A. C. Brańka, Phys. Lett. A 134, 314 (1988).

[15] H. Weber and D. Marx, Europhys. Lett. 27, 593 (1994).

[16] H. Weber, D. Marx, and K. Binder, Phys. Rev. B 51, 14636 (1995).

[17] J. A. Zollweg and G. V. Chester, Phys. Rev. B 46, 11186 (1992).

[18] A. Jaster, Phys. Rev. E 59, 2594 (1999).

[19] M. A. Bates and D. Frenkel, Phys. Rev. E 61, 5223 (2000).

[20] S. Sengupta, P. Nielaba, and K. Binder, Phys. Rev. E 61, 6294 
(2000)

[21] A. Jaster, Physica A 277, 106 (2000).

[22] A. Jaster, e-print cond-mat/0305239.

[23] P. G. Bolhuis and D. A. Kofke, Phys. Rev. E 54, 634 (1996).

[24] D. A. Kofke and P. G. Bolhuis, Phys. Rev. E 59, 618 (1999).

[25] M. R. Sadr-Lahijany, P. Ray, and H. E. Stanley, Phys. Rev. Lett. 79, 3206 (1997).

[26] L. Santen and W. Krauth, Nature (London) 405, 550 (2000).

[27] L. Santen and W. Krauth, e-print cond-mat/0107459.

[28] D. A. Kofke and E. D. Glandt, Mol. Phys. 64, 1105 (1988).

[29] H. B. Callen, Thermodynamics and an Introduction to Ther- mostatistics (Wiley, New York, 1985).

[30] D. C. Wallace, in Solid State Physics: Advances in Research and Applications, edited by H. Ehrenreich, F. Seitz, and D. Turnbull (Academic Press, New York, 1970), pp. 301-404.

[31] Y. Saito, Phys. Rev. B 26, 6239 (1982).

[32] S. T. Chui, Phys. Rev. B 28, 178 (1983).

[33] D. S. Fisher, B. I. Halperin, and R. Morf, Phys. Rev. B 20, 4692 (1979).

[34] P. Bladon and D. Frenkel, J. Phys. Chem. B 108, 6707 (2004).

[35] K. W. Wojciechowski, K. V. Tretiakov, A. C. Brańka, and M. Kowalik, J. Chem. Phys. 119, 939 (2003). 